Süleyman Demirel Üniversitesi Fen Edebiyat Fakültesi Fen Dergisi

Atıf için / For Citation: M. Yıldız, Ş. Altınkaya, "Sabordinasyon ve Fibonacci Sayılar Dizisi ile Tanımlanan Kendisi ve Tersi Yalınkat Fonksiyonların Yeni Bir Alt Sınıfı için Katsayı Eşitsizlikleri”, Süleyman Demirel Üniversitesi Fen Edebiyat Fakültesi Fen Dergisi, 16(1), 308-318, 2021.

\title{
Sabordinasyon ve Fibonacci Sayılar Dizisi ile Tanımlanan Kendisi ve Tersi Yalınkat Fonksiyonların Yeni Bir Alt Sınıfı için Katsayı Eşitsizlikleri
}

\author{
Meryem YILDIZ ${ }^{1}$, Şahsene ALTINKAYA*2 \\ ${ }^{1}$ Bursa Uludağ Üniversitesi, Fen Edebiyat Fakültesi, Matematik Bölümü, 16059, Bursa, Türkiye \\ ${ }^{2}$ Beykent Üniversitesi, Fen Edebiyat Fakültesi, Matematik Bölümü, 34500, İstanbul, Türkiye \\ *yazlşllan yazar e-posta: sahsenealtinkaya@beykent.edu.tr
}

(Alinış / Received: 21.05.2020, Kabul / Accepted: 05.12.2020, Yaylmlanma / Published: 27.05.2021)

Özet: $\mathrm{Bu}$ çalışmada, geometrik fonksiyonlar teorisinin en önemli araştırma konularından biri olan ve son y1llarda oldukça popüler hale gelen kendisi ve tersi yalınkat fonksiyonlar araştırılmıştır. Fonksiyonlar üzerine yapılan araştırmalarda sınır belirleme çalışması alışılagelen bir durumdur. $\mathrm{Bu}$ bağlamda, ilk olarak kendisi ve tersi yalınkat fonksiyonlar sınıfının $\mathbb{D}=$ $\{z \in \mathbb{C}:|z|<1\}$ açık birim diskinde yeni bir alt sınıfı tanımlanmıştır. Bu alt sınıf tanımlanırken kompleks değerli fonksiyonlar için geliştirilen Komatu integral operatörü ve sabordinasyon prensibi kullanılmıştır. Daha sonra Fibonacci sayı dizisi ile reel kısmı pozitif olan fonksiyonlar arasındaki ilişki verilmiştir. Bu ilişki Bulgular bölümü için temel teşkil etmektedir. Tanımlanan sınıfa ait fonksiyonların ilk iki Taylor Maclaurin katsayıları $a_{2}$ ve $a_{3}$ ün modülleri için üst sınırlar araştırılmıştır. Son olarak yine bu sınıfa ait fonksiyonlar için Fekete-Szegö eşitsizlikleri elde edilmiştir. Elde edilen bulgular literatürdeki sonuçlar ile karşılaştırılmıştır.

Anahtar kelimeler: Kendisi ve tersi yalınkat fonksiyonlar, Yıldızıl ve konveks fonksiyonlar, Katsayı eşitsizlikleri, Sabordinasyon, Komatu integral operatörü

\section{Coefficient Inequalities for Bi-univalent Functions Defined by Subordination and A Sequence of Fibonacci Numbers}

\begin{abstract}
In this work, bi-univalent functions, which are one of the most important research areas of Geometric Function Theory and which are still very popular in recent years, have been investigated. For the studies of functions, it is customary to determine the bound. In this direction, firstly, we introduce a new subclass of bi-univalent functions in the open unit disk $\mathbb{D}=\{z \in \mathbb{C}:|z|<1\}$. For this purpose, the Komatu integral operator developed for complex functions and subordination princible are used. Afterwards, the relation between the Fibonacci number sequence and the functions with the positive real part is given. This relation is a fundamental role for Results section. The upper bounds of the first two Taylor-Maclaurin coefficients are investigated. Finally, we derive Fekete-Szegö inequalities for functions belonging to this newly-defined class. The obtained results are compared with studies in the literature.
\end{abstract}

Key words: Bi-univalent functions, Starlike and convex functions, Coefficient inequalities, Subordination, Komatu integral operator 


\section{Giriş}

Kompleks düzlemin bir $B$ bölgesinde $f: B \rightarrow \mathbb{C}$ fonksiyonu birebir ise $f$ fonksiyonu $B$ de yalınkat fonksiyon olarak tanımlanmaktadır. Geometrik olarak ise düzlemde $f(B)$ görüntü bölgesinin katlı bölge olmaması şeklinde yorumlanmaktadır. $\mathbb{D}$ birim diskinde analitik ve $f(0)=f^{\prime}(0)-1=0$ koşuluyla normalize edilmiş fonksiyonların sınıfı $\mathcal{A}$ ile gösterilmek üzere her $f \in \mathcal{A}$ fonksiyonu

$$
f(z)=z+a_{2} z^{2}+a_{3} z^{3}+\ldots=z+\sum_{n=2}^{\infty} a_{n} z^{n}
$$

şeklinde bir Taylor serisi ile ifade edilebilir. $\mathbb{D}$ birim diskinde normalize edilmiş yalınkat analitik fonksiyonların sınıfı $\mathcal{S}$ ile gösterilir. $\mathcal{S}$ sınıfında çalışılan temel problemler yeni alt sınıflar tanımlama, katsayı sınırları, Fekete-Szegö eşitsizlikleri, Hankel determinant sınırları, büyüme-genişleme teoremleri, integral ve türev operatörleri, konvolüsyon ve sabordinasyon teknikleridir.

$\mathcal{S}$ sınıfının iki önemli alt sınıfı $\alpha$ mertebeli yıldızıl fonksiyonların sınıfı $\mathcal{S}^{*}(\alpha)$ ve $\alpha$ mertebeli konveks fonksiyonların sınıfı $K(\alpha)$ dır. Matematiksel olarak

$$
\mathcal{S}^{*}(\alpha)=\left\{f: f \in \mathcal{S} \text { and } \mathfrak{R}\left(\frac{z f^{\prime}(z)}{f(z)}\right)>\alpha, \quad(0 \leq \alpha<1, z \in \mathbb{D})\right\}
$$

ve

$$
K(\alpha)=\left\{f: f \in \mathcal{S} \text { and } \mathfrak{R}\left(1+\frac{z f^{\prime \prime}(z)}{f^{\prime}(z)}\right)>\alpha, \quad(0 \leq \alpha<1, z \in \mathbb{D})\right\}
$$

biçiminde ifade edilmektedir.

$\mathbb{D}$ açık birim diskinde analitik, $p(0)=1$ ve her $z \in \mathbb{D}$ için $\Re p(z)>0$ koşullarını sağlayan fonksiyonlar reel kısmı pozitif analitik fonksiyonlar olarak tanımlanmaktadır ve bu fonksiyonların sınıfı $\mathcal{P}$ ile gösterilmektedir. Literatürde Carathéodory sınıfı olarak da bilinmektedir. Ayrıca bir $p \in \mathcal{P}$ fonksiyonu

$$
p(z)=1+x_{1} z+x_{2} z^{2}+x_{3} z^{3}+\cdots
$$

formunda seri açılımına sahiptir ve katsayıları için $\left|x_{n}\right| \leq 2 \quad(n \in \mathbb{N}=\{1,2,3, \ldots\})$ eşitsizliği sağlanmaktadır $[13,22]$.

Şimdi, kompleks analizde önemli rol oynayan sabordinasyon tanımı verilecektir.

Bir $\mathfrak{w}$ Schwarz fonksiyonu

$$
\mathfrak{w}(z)=\sum_{n=1}^{\infty} c_{n} z^{n} \quad(\mathfrak{w}(0)=0,|\mathfrak{w}(z)|<1)
$$

formunda tanımlı olmak üzere $\mathbb{D}$ açık birim diskinde tanımlı analitik $f$ ve $g$ fonksiyonları 


$$
f(z)=g(\mathfrak{w}(z)) \quad(z \in \mathbb{D})
$$

şeklinde ifade edilebiliyorsa $f$ fonksiyonu $g$ fonksiyonuna sabordinedir denir ve $f(z) \prec g(z)$ şeklinde gösterilir. Burada ise $\mathfrak{w}(z)$ Schwarz fonksiyonu için $\left|c_{n}\right|<1$ katsayı eşitsizliği bilinmektedir [11].

$\mathcal{S}$ sinıfina ait Denklem 1 ile verilen bir $f \in \mathcal{A}$ fonksiyonu

$$
f^{-1}(f(z))=z \quad(z \in \mathbb{D})
$$

ve

$$
f\left(f^{-1}(w)\right)=w\left(|w|<r_{0}(f), r_{0}(f) \geq \frac{1}{4}\right)
$$

eşitliklerini sağlayan

$$
g(w)=f^{-1}(w)=w-a_{2} w^{2}+\left(2 a_{2}^{2}-a_{3}\right) w^{3}-\left(5 a_{2}^{3}-5 a_{2} a_{3}+a_{4}\right) w^{4}+\cdots
$$

biçiminde bir $f^{-1}$ ters fonksiyonuna sahiptir [11]. Eğer hem $f$ hem de $f^{-1}$ fonksiyonu $\mathbb{D}$ birim diskinde yalınkat ise $f$ fonksiyonuna kendisi ve tersi yalınkat (bi-univalent) fonksiyon denir. Kendisi ve tersi yalınkat fonksiyonların sınıfı $\Sigma$ ile gösterilir. $\Sigma$ sınıfına ait bazı fonksiyonlar

$$
h(z)=\frac{z}{1-z}, g(z)=-\log (1-z), \quad k(z)=\frac{1}{2} \log \left(\frac{1+z}{1-z}\right)
$$

olarak verilebilir.

1967 yılında Lewin, tersi de yalınkat olan fonksiyonları kendisi ve tersi yalınkat (biunivalent) fonksiyonlar olarak adlandırmış ve bu fonksiyonların oluşturduğu sınıfı $\Sigma$ ile göstermiştir [18]. $\Sigma$ sınıfına ait fonksiyonlar için katsayı sınırları elde etme çalışmaları yapmıştır ve $\left|a_{2}\right| \leq 1,51$ sınırını elde etmiştir. 1986 yılında Brannan ve Taha kendisi ve tersi yalınkat fonksiyonların bazı alt sınıflarının başlangıç katsayıları için tahminler elde etmiştir [9]. Son yıllarda yapılan çalışmalarda bu problem tekrar çalışılmaya başlanmış [25]; $\Sigma$ sınıfı ve Taylor-Maclaurin serilerinin ilk iki katsayısı olan $a_{2}$ ve $a_{3}$ ün modülleri için üst sınırları bulma çalışmaları popüler hale gelmiştir $[2,3,4,5,6,15,21]$. Literatürde $a_{n}$ in modülü için üst sınırların elde edildiği çalışmalar olmasına rağmen $[8,24,26] n \in \mathbb{N} \backslash\{1,2\}$ için $\left|a_{n}\right|$ genel katsayı tahmini hala açı bir problemdir.

Sabordinasyon tanımı ve

$$
\frac{z f^{\prime}(z)}{f(z)}, \quad 1+\frac{z f^{\prime \prime}(z)}{f^{\prime}(z)}
$$

denklemleri kullanılarak yıldızıl ve konveks fonksiyonlar için çalışmalar yapılmıştır [20]. İlk olarak sabordinasyon tanımı gereği $\mathbb{D}$ birim diskinde reel kısmı pozitif ve reel eksene göre simetrik, 1 noktasına göre y1ldızıl bir bölgeye resmeden analitik bir $\varphi(\varphi$ $(0)=0, \varphi^{\prime}(0)=1$ ) fonksiyonu göz önüne alınmıştır. Böylece Ma-Minda yıldızıl fonksiyonlar sinıfı 


$$
\frac{z f^{\prime}(z)}{f(z)} \prec \varphi(z)
$$

sabordinasyon şartını sağlayan $f \in \mathcal{A}$ fonksiyonlarından oluşmaktadır. Benzer şekilde, Ma-Minda konveks fonksiyonlar sinıfi

$$
1+\frac{z f^{\prime \prime}(z)}{f^{\prime}(z)} \prec \varphi(z)
$$

sabordinasyon şartını sağlayan $f \in \mathcal{A}$ fonksiyonlarından oluşmaktadır. $\mathrm{Bu}$ tanımlamalardan yola çıkılarak hem $f$ hem de $f^{-1}$ fonksiyonu Ma-Minda yıldızıl ise $f$ fonksiyonunu Ma-Minda bi-yıldızıl, hem $f$ hem de $f^{-1}$ fonksiyonu Ma-Minda konveks ise $f$ fonksiyonunu Ma-Minda bi-konveks olarak adlandırılmıştır [11]. Ma-Minda biyıldızıl ve Ma-Minda bi-konveks fonksiyonların sinıfi sirasiyla $\mathcal{S}^{*}(\varphi), K(\varphi)$ ile gösterilmektedir [7].

Kompleks analizin ilgilendiği önemli problemlerden olan Fekete-Szegö problemi

$$
F_{\varrho}(f)=a_{3}-\varrho a_{2}^{2}
$$

fonksiyonu için üst sınır elde etmek olarak bilinmektedir [12]. $f \in \mathcal{S}$ için Fekete-Szegö eşitsizlikleri

$$
\left|a_{3}-\varrho a_{2}^{2}\right| \leq 1+2 \exp \left(-\frac{2 \varrho}{1-\vartheta}\right) \quad \varrho \in[0,1)
$$

şeklinde elde edilmektedir.

\section{Materyal ve Metot}

$\mathrm{Bu}$ bölümde Komatu integral operatörü, kendisi ve tersi yalınkat fonksiyonların $\mathcal{S} \mathcal{L}_{\mathrm{t}}^{\mu}(\tilde{\mathrm{p}} ; \varepsilon)$ alt sınıfı tanımlanmıştır ve Fibonacci sayıları hakkında bazı ifadeler verilmiştir.

Tanım 2.1. Komatu integral operatorü $\mathcal{L}_{t}^{\mu}$

$$
\mathcal{L}_{t}^{\mu} f(z)=\frac{t^{\mu}}{\Gamma(\mu)} \int_{0}^{1} \zeta^{t-2} \log \left(\frac{1}{\zeta}\right)^{\mu-1} f(z \zeta) d \zeta \quad(t>0, \mu \geq 0, f \in \mathcal{A}, z \in \mathbb{D})
$$

şeklinde tanımlanmaktadır [17]. Kompleks değerli, Denklem 1 formundaki bir $f \in \mathcal{A}$ fonksiyonu için Komatu integral operatorü $\mathcal{L}_{t}^{\mu} f(z)$

$$
\mathcal{L}_{t}^{\mu} f(z)=z+\sum_{n=2}^{\infty}\left(\frac{t}{t+n-1}\right)^{\mu} a_{n} z^{n} \quad(t>0, \mu \geq 0, z \in \mathbb{D})
$$

şeklinde tanımlanır.

Komatu integral operatörü tanımı gereği $\mathcal{L}_{t}^{\mu}\left(z f^{\prime}(z)\right)=z\left(\mathcal{L}_{t}^{\mu} f(z)\right)^{\prime}$ yazılabilir. Ayrıca $\mu$ ve $t$ değişkenlerinin bazı özel değerleri için Komatu integral operatörü $\mathcal{L}_{t}^{\mu} f(z)$ aşağıdaki operatörlere dönüşür: 
- $\mathcal{L}_{t}^{0} f(z)=f(z)$,

- $\quad \mathcal{L}_{1}^{1} f(z)=A[f](z)$ Alexander operatörü [1],

- $\quad \mathcal{L}_{2}^{1} f(z)=\mathcal{L}[f](z)$ Libera operatörü [19],

- $\mathcal{L}_{1}^{-k} f(z)=D^{k} f(z)\left(k \in \mathbb{N}_{0}\right)$ Sălăgean türev operatörü [23],

- $\mathcal{L}_{2}^{\mu} f(z)=I^{\mu} f(z)$ operatörü [16].

Şimdi sabordinasyon tanımı ve Komatu integral operatörünü kullanarak kendisi ve tersi yalınkat fonksiyonların $\mathcal{S} \mathcal{L}_{\mathrm{t}}^{\mu}(\tilde{\mathrm{p}} ; \varepsilon)$ alt sınıfı tanımlanacaktır.

Tanım 2.2. Kendisi ve tersi yalınkat fonksiyonların $\Sigma$ sinıfina ait bir $f$ fonksiyonu $0 \leq \varepsilon \leq 1, t>0, \mu \geq 0$ ve $\tau=\frac{1-\sqrt{5}}{2} \approx-0.618$ olmak üzere

$$
\frac{z\left(\varepsilon z\left(\mathcal{L}_{t}^{\mu} f(z)\right)^{\prime}+(1-\varepsilon) \mathcal{L}_{t}^{\mu} f(z)\right)^{\prime}}{\varepsilon z\left(\mathcal{L}_{t}^{\mu} f(z)\right)^{\prime}+(1-\varepsilon) \mathcal{L}_{t}^{\mu} f(z)} \prec \tilde{\mathrm{p}}(z)=\frac{1+\tau^{2} z^{2}}{1-\tau z-\tau^{2} z^{2}} \quad(z \in \mathbb{D})
$$

ve

$$
\frac{w\left(\varepsilon w\left(\mathcal{L}_{t}^{\mu} g(w)\right)^{\prime}+(1-\varepsilon) \mathcal{L}_{t}^{\mu} g(w)\right)^{\prime}}{\varepsilon w\left(\mathcal{L}_{t}^{\mu} g(w)\right)^{\prime}+(1-\varepsilon) \mathcal{L}_{t}^{\mu} g(w)} \prec \tilde{\mathrm{p}}(\mathrm{w})=\frac{1+\tau^{2} w^{2}}{1-\tau w-\tau^{2} w^{2}} \quad(w \in \mathbb{D})
$$

sabordinasyon şartlarını sağlıyorsa $\mathcal{S} \mathcal{L}_{\mathrm{t}}^{\mu}(\tilde{\mathrm{p}} ; \varepsilon)$ sınıfındandır denir.

Uyarı 2.3. Eğer $\tilde{\mathrm{p}}(0)=\tilde{\mathrm{p}}\left(-\frac{1}{2 \tau}\right)$ ve $\tilde{\mathrm{p}}\left(e^{ \pm i \arccos (1 / 4)}\right)=\frac{\sqrt{5}}{5}$ değerleri göz önünde bulundurulursa $\tilde{\mathrm{p}}(z)$ fonksiyonunun $\mathbb{D}$ birim diskinde yalınkat olmadığı sonucuna ulaşı1ır. Dolayısıyla $\tilde{\mathrm{p}}(z)$ fonksiyonunun yalınkat olduğu diskin belirlenmesi gerekmektedir ve $\tilde{\mathrm{p}}(z)$ fonksiyonunun yalınkat olduğu disk $|z|<\frac{3-\sqrt{5}}{2} \approx 0.38$ olarak bulunmuştur [10]. Bu sonuç ise

$$
\frac{1}{|\tau|}=\frac{|\tau|}{1-|\tau|}
$$

eşitliğini vermektedir. Burada $|\tau|,[0,1]$ aralığını altın oranı karşılayacak şekilde ayırdığı sonucuna ulaşılmıştır. Ayrıca, Fibonacci sayıları ve $\tilde{\mathrm{p}}(z)$ fonksiyonu arasında aşağıdaki bağlantı elde edilmiştir [10]:

$\left\{\mathrm{F}_{n}\right\}$ Fibonacci sayılarının bir dizisi ve

$$
\mathrm{F}_{0}=0, \mathrm{~F}_{1}=1, \mathrm{~F}_{n+2}=\mathrm{F}_{n}+\mathrm{F}_{n+1} \quad\left(n \in \mathbb{N}_{0}=\mathbb{N} \cup\{0\}\right)
$$

ise

$$
\mathrm{F}_{n}=\frac{(1-\tau)^{n}-\tau^{n}}{\sqrt{5}}, \quad \tau=\frac{1-\sqrt{5}}{2}
$$


dir. Bu durumda $\tilde{\mathrm{p}}(z)$ fonksiyonu

$$
\begin{aligned}
\tilde{\mathrm{p}}(z)=1 & +\sum_{n=1}^{\infty} \tilde{\mathrm{p}}_{n} z^{n}=1+\left(\mathrm{F}_{0}+\mathrm{F}_{2}\right) \tau z+\left(\mathrm{F}_{1}+\mathrm{F}_{3}\right) \tau^{2} z^{2} \\
& +\sum_{n=3}^{\infty}\left(\mathrm{F}_{n-3}+\mathrm{F}_{n-2}+\mathrm{F}_{n-1}+\mathrm{F}_{n}\right) \tau^{n} z^{n}
\end{aligned}
$$

olarak ele alınırsa, $\tilde{\mathrm{p}}_{n}$ katsayıları için

$$
\tilde{\mathrm{p}}_{n}=\left\{\begin{array}{cl}
\tau & (n=1) \\
3 \tau^{2} & (n=2) \\
\tau \tilde{\mathrm{p}}_{n-1}+\tau^{2} \tilde{\mathrm{p}}_{n-2} & (n=3,4, \cdots)
\end{array}\right.
$$

bulunur.

Şimdi $\varepsilon=0$ ve $\varepsilon=1$ olması durumunda elde edilen ve çalışılan fonksiyon sınıfları $\mathcal{S} \mathcal{L}_{\Sigma}(\tilde{\mathrm{p}}(z))$ ve $\mathcal{S} \mathcal{L}_{\Sigma}(\tilde{\mathrm{p}}(z))$ verilecektir.

Sonuç 2.4. Kendisi ve tersi yalınkat fonksiyonların $\Sigma$ sınıfına ait bir $f$ fonksiyonu $t>0, \mu \geq 0$ ve $\tau=\frac{1-\sqrt{5}}{2} \approx-0.618$ olmak üzere

$$
\frac{z\left(\mathcal{L}_{t}^{\mu} f(z)\right)^{\prime}}{\mathcal{L}_{t}^{\mu} f(z)} \prec \tilde{\mathrm{p}}(z)=\frac{1+\tau^{2} z^{2}}{1-\tau z-\tau^{2} z^{2}} \quad(z \in \mathbb{D})
$$

ve

$$
\frac{w\left(\mathcal{L}_{t}^{\mu} g(w)\right)^{\prime}}{\mathcal{L}_{t}^{\mu} g(w)} \prec \tilde{\mathrm{p}}(\mathrm{w})=\frac{1+\tau^{2} w^{2}}{1-\tau w-\tau^{2} w^{2}} \quad(w \in \mathbb{D})
$$

sabordinasyon şartlarını sağlıyorsa $\mathcal{S} \mathcal{L}_{\Sigma}(\tilde{\mathrm{p}}(z))$ sınıfındandır denir [14].

Sonuç 2.5. Kendisi ve tersi yalınkat fonksiyonların $\Sigma$ sınıfına ait bir $f$ fonksiyonu $t>0, \mu \geq 0$ ve $\tau=\frac{1-\sqrt{5}}{2} \approx-0.618$ olmak üzere

$$
\frac{z\left(z\left(\mathcal{L}_{t}^{\mu} f(z)\right)^{\prime}\right)^{\prime}}{z\left(\mathcal{L}_{t}^{\mu} f(z)\right)^{\prime}}<\tilde{\mathrm{p}}(z)=\frac{1+\tau^{2} z^{2}}{1-\tau z-\tau^{2} z^{2}} \quad(z \in \mathbb{D})
$$

ve

$$
\frac{w\left(w\left(\mathcal{L}_{t}^{\mu} g(w)\right)^{\prime}\right)^{\prime}}{w\left(\mathcal{L}_{t}^{\mu} g(w)\right)^{\prime}} \prec \tilde{\mathrm{p}}(\mathrm{w})=\frac{1+\tau^{2} w^{2}}{1-\tau w-\tau^{2} w^{2}} \quad(w \in \mathbb{D})
$$


sabordinasyon şartlarını sağlıyorsa $K \mathcal{L}_{\Sigma}(\tilde{\mathrm{p}}(z))$ sınıfındandır denir [14].

\section{Bulgular}

$\mathrm{Bu}$ bölümde, $\mathcal{S} \mathcal{L}_{\mathrm{t}}^{\mu}(\tilde{\mathrm{p}} ; \varepsilon)$ sınıfına ait fonksiyonların başlangıç katsayıları $a_{2}$ ve $a_{3}$ ün modülleri için üst sınırlar ve Fekete-Szegö eşitsizlikleri elde edilmiştir.

Teorem 3.1. Denklem 1 ile ifade edilen $f$ fonksiyonu $\mathcal{S} \mathcal{L}_{\mathrm{t}}^{\mu}(\tilde{\mathrm{p}} ; \varepsilon)$ sinıfina ait olsun. Bu durumda

$$
\begin{gathered}
\left|a_{2}\right| \leq \frac{|\tau|}{\sqrt{\left|\left[2(1+2 \varepsilon)\left(\frac{t}{t+2}\right)^{\mu}-4(1+\varepsilon)^{2}\left(\frac{t}{t+1}\right)^{2 \mu}\right] \tau+(1+\varepsilon)^{2}\left(\frac{t}{t+1}\right)^{2 \mu}\right|}}, \\
\left|a_{3}\right| \leq \frac{|\tau|}{2(1+2 \varepsilon)\left(\frac{t}{t+2}\right)^{\mu}}+\frac{\tau^{2}}{(1+\varepsilon)^{2}\left(\frac{t}{t+1}\right)^{2 \mu}}
\end{gathered}
$$

eşitsizlikleri elde edilir. Herhangi bir $\varrho$ reel sayısı için Fekete-Szegö eşitsizlikleri ise

$$
M=\frac{\left|\left[2(1+2 \varepsilon)\left(\frac{t}{t+2}\right)^{\mu}-4(1+\varepsilon)^{2}\left(\frac{t}{t+1}\right)^{2 \mu}\right] \tau+(1+\varepsilon)^{2}\left(\frac{t}{t+1}\right)^{2 \mu}\right|}{2|\tau|(1+2 \varepsilon)\left(\frac{t}{t+2}\right)^{\mu}}
$$

olmak üzere

$\left|a_{3}-\varrho a_{2}^{2}\right| \leq$

$$
\left\{\begin{array}{cc}
\frac{|\tau|}{2(1+2 \varepsilon)\left(\frac{t}{t+2}\right)^{\mu}}, & |1-\varrho| \leq M \\
\frac{|1-\varrho| \tau^{2}}{\left[2(1+2 \varepsilon)\left(\frac{t}{t+2}\right)^{\mu}-4(1+\varepsilon)^{2}\left(\frac{t}{t+1}\right)^{2 \mu}\right] \tau+(1+\varepsilon)^{2}\left(\frac{t}{t+1}\right)^{2 \mu}}, & |1-\varrho| \geq M
\end{array}\right.
$$

şeklindedir.

İspat. Kabul edelim ki $f \in \mathcal{S} \mathcal{L}_{\mathrm{t}}^{\mu}(\tilde{\mathrm{p}} ; \varepsilon)$ ve $\mathrm{p} \prec \tilde{\mathrm{p}}$ olsun. Sabordinasyon tanımı gereği analitik $\mathrm{u}(\mathrm{u}(0)=0,|\mathrm{u}(z)|<1)$ ve $\mathrm{v}(\mathrm{v}(0)=0,|\mathrm{v}(w)|<1)$ fonksiyonları için

$$
\frac{z\left(\varepsilon z\left(\mathcal{L}_{t}^{\mu} f(z)\right)^{\prime}+(1-\varepsilon) \mathcal{L}_{t}^{\mu} f(z)\right)^{\prime}}{\varepsilon z\left(\mathcal{L}_{t}^{\mu} f(z)\right)^{\prime}+(1-\varepsilon) \mathcal{L}_{t}^{\mu} f(z)}=\tilde{\mathrm{p}}(\mathrm{u}(z))
$$

ve 


$$
\frac{w\left(\varepsilon w\left(\mathcal{L}_{t}^{\mu} g(w)\right)^{\prime}+(1-\varepsilon) \mathcal{L}_{t}^{\mu} g(w)\right)^{\prime}}{\varepsilon w\left(\mathcal{L}_{t}^{\mu} g(w)\right)^{\prime}+(1-\varepsilon) \mathcal{L}_{t}^{\mu} g(w)}=\tilde{\mathrm{p}}(\mathrm{v}(w))
$$

eşitlikleri yazılabilir. Burada $p_{1}, p_{2} \in \mathcal{P}$ olmak üzere

$$
\begin{gathered}
p_{1}(z)=\frac{1+\mathrm{u}(z)}{1-\mathrm{u}(z)}=1+x_{1} z+x_{2} z^{2}+\cdots \\
p_{2}(w)=\frac{1+\mathrm{v}(w)}{1-\mathrm{v}(w)}=1+y_{1} w+y_{2} w^{2}+\cdots
\end{gathered}
$$

fonksiyonları ve u, $\mathrm{v}$ Schwarz fonksiyonları

$$
\begin{aligned}
& \mathrm{u}(z)=\frac{p_{1}(z)-1}{p_{1}(z)+1}=\frac{1}{2}\left[x_{1} z+\left(x_{2}-\frac{x_{1}^{2}}{2}\right) z^{2}\right]+\cdots, \\
& \mathrm{v}(w)=\frac{p_{2}(w)-1}{p_{2}(w)+1}=\frac{1}{2}\left[y_{1} w+\left(y_{2}-\frac{y_{1}^{2}}{2}\right) w^{2}\right]+\cdots
\end{aligned}
$$

olarak tanımlanmıştır. Dolayısıyla

$$
\begin{aligned}
& \tilde{\mathrm{p}}(\mathrm{u}(z))=1+\frac{\tilde{\mathrm{p}}_{1} x_{1}}{2} z+\left[\frac{1}{2}\left(x_{2}-\frac{x_{1}^{2}}{2}\right) \tilde{\mathrm{p}}_{1}+\frac{x_{1}^{2}}{4} \tilde{\mathrm{p}}_{2}\right] z^{2}+\cdots, \\
& \tilde{\mathrm{p}}(\mathrm{v}(\mathrm{w}))=1+\frac{\tilde{\mathrm{p}}_{1} y_{1}}{2} w+\left[\frac{1}{2}\left(y_{2}-\frac{y_{1}^{2}}{2}\right) \tilde{\mathrm{p}}_{1}+\frac{y_{1}^{2}}{4} \tilde{\mathrm{p}}_{2}\right] w^{2}+\cdots
\end{aligned}
$$

olduğu kolayca elde edilir. Elde edilen bu bulgular Denklem 4 ve Denklem 5 ile birlikte düşünülürse

$$
\begin{gathered}
(1+\varepsilon)\left(\frac{t}{t+1}\right)^{\mu} a_{2}=\frac{\tilde{\mathrm{p}}_{1} x_{1}}{2} \\
2(1+2 \varepsilon)\left(\frac{t}{t+2}\right)^{\mu} a_{3}-(1+\varepsilon)^{2}\left(\frac{t}{t+1}\right)^{2 \mu} a_{2}^{2}=\frac{1}{2}\left(x_{2}-\frac{x_{1}^{2}}{2}\right) \tilde{\mathrm{p}}_{1}+\frac{x_{1}^{2}}{4} \tilde{\mathrm{p}}_{2}
\end{gathered}
$$

ve

$$
\begin{gathered}
-(1+\varepsilon)\left(\frac{t}{t+1}\right)^{\mu} a_{2}=\frac{\tilde{\mathrm{p}}_{1} y_{1}}{2}, \\
2(1+2 \varepsilon)\left(\frac{t}{t+2}\right)^{\mu}\left(2 a_{2}^{2}-a_{3}\right)-(1+\varepsilon)^{2}\left(\frac{t}{t+1}\right)^{2 \mu} a_{2}^{2}=\frac{1}{2}\left(y_{2}-\frac{y_{1}^{2}}{2}\right) \tilde{\mathrm{p}}_{1}+\frac{y_{1}^{2}}{4} \tilde{\mathrm{p}}_{2}
\end{gathered}
$$


bulunur. Böylece, Denklem 6 ve Denklem 8

$$
\begin{gathered}
x_{1}=-y_{1} \\
2(1+\varepsilon)^{2}\left(\frac{t}{t+1}\right)^{2 \mu} a_{2}^{2}=\frac{\tilde{\mathrm{p}}_{1}^{2}}{4}\left(x_{1}^{2}+y_{1}^{2}\right)
\end{gathered}
$$

eşitliklerini verir. Denklem 7 ve Denklem 9 taraf tarafa toplandığında ise

$$
2\left[2(1+2 \varepsilon)\left(\frac{t}{t+2}\right)^{\mu}-(1+\varepsilon)^{2}\left(\frac{t}{t+1}\right)^{2 \mu}\right] a_{2}^{2}=\frac{\tilde{\mathrm{p}}_{1}}{2}\left(x_{2}+y_{2}\right)+\frac{\left(\tilde{\mathrm{p}}_{2}-\tilde{\mathrm{p}}_{1}\right)}{4}\left(x_{1}^{2}+y_{1}^{2}\right)
$$

eşitliği ve buradan elde edilen

$$
a_{2}^{2}=\frac{\widetilde{\mathrm{p}}_{1}^{3}\left(x_{2}+y_{2}\right)}{4\left\{\left[2(1+2 \varepsilon)\left(\frac{t}{t+2}\right)^{\mu}-(1+\varepsilon)^{2}\left(\frac{t}{t+1}\right)^{2 \mu}\right] \widetilde{\mathrm{p}}_{1}^{2}-\left(\widetilde{\mathrm{p}}_{2}-\widetilde{\mathrm{p}}_{1}\right)(1+\varepsilon)^{2}\left(\frac{t}{t+1}\right)^{2 \mu}\right\}}
$$

denklemine üçgen eşitsizliği uygulanırsa ve Denklem 3 göz önüne alınırsa başlangıç katsayısı $a_{2}$ modülü için üst sınır

$$
\left|a_{2}\right| \leq \frac{|\tau|}{\sqrt{\left|\left[2(1+2 \varepsilon)\left(\frac{t}{t+2}\right)^{\mu}-4(1+\varepsilon)^{2}\left(\frac{t}{t+1}\right)^{2 \mu}\right] \tau+(1+\varepsilon)^{2}\left(\frac{t}{t+1}\right)^{2 \mu}\right|}}
$$

olarak bulunur.

Diğer yandan, Denklem 7 ve Denklem 9 taraf tarafa çıkarıldığında

$$
4(1+2 \varepsilon)\left(\frac{t}{t+2}\right)^{\mu}\left(a_{3}-a_{2}^{2}\right)=\frac{\tilde{p}_{1}}{2}\left(x_{2}-y_{2}\right)
$$

ve buradan

$$
a_{3}=\frac{\tilde{\mathrm{p}}_{1}\left(x_{2}-y_{2}\right)}{8(1+2 \varepsilon)\left(\frac{t}{t+2}\right)^{\mu}}+\frac{\tilde{\mathrm{p}}_{1}^{2}\left(x_{1}^{2}+y_{1}^{2}\right)}{8(1+\varepsilon)^{2}\left(\frac{t}{t+1}\right)^{2 \mu}}
$$

elde edilir. Şimdi üçgen eşitsizliği ve Denklem 3 kullanılırsa $\left|a_{3}\right|$ için istenen üst sınıra kolayca ulaşı1ır.

$\mathcal{S} \mathcal{L}_{\mathrm{t}}^{\mu}(\tilde{\mathrm{p}} ; \varepsilon)$ sınıfına ait fonksiyonların Fekete-Szegö eşitsizliklerini elde etmek için Denklem 13 ve Denklem 14 kullanılırsa

$$
a_{3}-\varrho a_{2}^{2}=\frac{(1-\varrho) \widetilde{\mathrm{p}}_{1}^{3}\left(x_{2}+y_{2}\right)}{4\left\{\left[2(1+2 \varepsilon)\left(\frac{t}{t+2}\right)^{\mu}-(1+\varepsilon)^{2}\left(\frac{t}{t+1}\right)^{2 \mu}\right] \widetilde{\mathrm{p}}_{1}^{2}-\left(\widetilde{\mathrm{p}}_{2}-\widetilde{\mathrm{p}}_{1}\right)(1+\varepsilon)^{2}\left(\frac{t}{t+1}\right)^{2 \mu}\right\}}+\frac{\tilde{\mathrm{p}}_{1}\left(x_{2}-y_{2}\right)}{8(1+2 \varepsilon)\left(\frac{t}{t+2}\right)^{\mu}}
$$




$$
=\frac{\tilde{p}_{1}}{4}\left[\left(h(\varrho)+\frac{1}{2(1+2 \varepsilon)\left(\frac{t}{t+2}\right)^{\mu}}\right) x_{2}+\left(h(\varrho)-\frac{1}{2(1+2 \varepsilon)\left(\frac{t}{t+2}\right)^{\mu}}\right) y_{2}\right]
$$

bulunur. Burada

$$
h(\varrho)=\frac{(1-\varrho) \tilde{\mathrm{p}}_{1}{ }^{2}}{\left[2(1+2 \varepsilon)\left(\frac{t}{t+2}\right)^{\mu}-(1+\varepsilon)^{2}\left(\frac{t}{t+1}\right)^{2 \mu}\right] \tilde{\mathrm{p}}_{1}^{2}-\left(\tilde{\mathrm{p}}_{2}-\tilde{\mathrm{p}}_{1}\right)(1+\varepsilon)^{2}\left(\frac{t}{t+1}\right)^{2 \mu}}
$$

olarak ele alınmıştır. Üçgen eşitsizliği uygulandıktan sonra Denklem 3 göz önüne alınırsa Fekete-Szegö eşitsizlikleri

$$
\left|a_{3}-\varrho a_{2}^{2}\right| \leq\left\{\begin{array}{cl}
\frac{\left|\tilde{\mathrm{p}}_{1}\right|}{2(1+2 \varepsilon)\left(\frac{t}{t+2}\right)^{\mu}}, & 0 \leq|h(\varrho)| \leq \frac{1}{2(1+2 \varepsilon)\left(\frac{t}{t+2}\right)^{\mu}} \\
\left|\tilde{\mathrm{p}}_{1}\right||h(\varrho)|, & |h(\varrho)| \geq \frac{|\tau|}{2(1+2 \varepsilon)\left(\frac{t}{t+2}\right)^{\mu}}
\end{array}\right.
$$

olarak elde edilir.

\section{Sonuç ve Yorum}

Literatürde kendisi ve tersi yalınkat fonksiyonların birçok alt sınıfı için katsayı eşitsizlikleri araştırılmıştır. Bu araştırmalardan farklı olarak, bu çalışmada, Fibonacci sayı dizileri, sabordinasyon ve Komatu integral operatörü kullanılarak kendisi ve tersi yalınkat fonksiyonların

$$
\mathcal{S} \mathcal{L}_{\mathrm{t}}^{\mu}(\tilde{\mathrm{p}} ; \varepsilon) \quad(0 \leq \varepsilon \leq 1, t>0, \mu \geq 0)
$$

alt sınıfı tanımlanmıştır. $\mathrm{Bu}$ sınıfa ait fonksiyonlar için Taylor-Maclaurin katsayı eşitsizlikleri ve Fekete-Szegö eşitsizlikleri elde edilmiştir. $\mathcal{S} \mathcal{L}_{\mathrm{t}}^{\mu}(\tilde{\mathrm{p}} ; \varepsilon)$ sınıfının geometrik özellikleri $\varepsilon, t, \mu$ paremetrelerine bağlı olarak değişmektedir. $\mathrm{Bu}$ nedenle, ilgili parametrelerin özel durumları için bazı sonuçlar açıklayıcı örnekler olarak sunulabilir. $\mathrm{Bu}$ özel durumlar okuyucuya alıştırma olarak bırakılmıştır. Ayrıca, bu makalede üzerinde çalıştığımız fonksiyon sınıfının diğer birçok alt sınıfı tanımlanabilir.

\section{Araştırmacıların Katkı Oranı Beyanı}

Meryem Yıldız: Kaynak/Materyal/Malzeme Temini, Araştırma, Orijinal Taslak Yazımı. Şahsene Altınkaya: Araştırma, Doğrulama, İnceleme ve Düzenleme.

\section{Destek}

Bu çalışma, Şahsene ALTINKAYA'nın yürütücüsü, Meryem YILDIZ’ın bursiyeri olduğu TÜBİTAK 118F543 nolu proje ile desteklenmektedir.

\section{Çatışma Beyanı}

Bu çalışmanın yazarları olarak herhangi bir çatışma beyanımız bulunmadığını bildiririz.

\section{Etik Kurul Onayı ve/veya Aydınlatılmış Onam Bilgileri}

$\mathrm{Bu}$ çalışmanın yazarları olarak herhangi bir etik kurul onayı ve/veya aydınlatılmış onam bilgileri beyanımız bulunmadığını bildiririz. 


\section{Kaynakça}

[1] J. W. Alexander, "Function which map the interior of the unit circle upon simple regions," Ann. Math., Second Series, 17, 12-22, 1915.

[2] O. P. Ahuja, A. Çetinkaya, N. Bohra, "On a class of $q$-bi-univalent functions of complex order related to shell-like curves connected with the Fibonacci numbers," Honam Mathematical J., 42, 319-330, 2020.

[3] A. Akgül, " $(p, q)$-Lucas polynomial coefficient inequalities of the bi-univalent function class," Turk. J. Math., 43, 2170-2176, 2019.

[4] A. Akgül, F. M. Sakar, "A certain subclass of bi-univalent analytic functions introduced by means of the $q$-analogue of Noor integral operator and Horadam polynomials," Turk. J. Math., 43, 2275-2286, 2019.

[5] A. Akgül, "The Fekete-Szegö coefficient inequalitiy for a new class of $m$-fold symmetric biunivalent functions satisfying subordination condition," Honam Mathematical J., 70, 733-748, 2018.

[6] A. Akgül, "New subclasses of analytic and bi-univalent functions involving a new integral operator defined by polylogarithm function," Theory Appl. Math. Comput. Sci., 7, 31-40, 2017.

[7] R. M. Ali, S. K. Lee, V. Ravichandran, S. Supramanian, "Coefficient estimates for bi-univalent MaMinda starlike and convex functions," Appl. Math. Lett., 25, 344-351, 2012.

[8] Ş. Altınkaya, S. Yalçın, S. Çakmak, "A Subclass of bi-univalent functions based on the Faber polynomial expansions and the Fibonacci numbers," Mathematics, 7, 1-9, 2019.

[9] D. A. Brannan, T. S. Taha, "On some classes of bi-univalent functions," Stud. Univ. Babeş-Bolyai Math., 31, 70-77, 1986.

[10] J. Dziok, R. K. Raina, J. Sokól, “On $\alpha$-convex functions related to shell-like functions connected with Fibonacci numbers,” Appl. Math. Comput., 218, 996-1002, 2011.

[11] P. L. Duren, Univalent Functions, Grundlehren der Mathematischen Wissenschaften, Springer, New York, 1983.

[12] M. Fekete, G. Szegö, "Eine bemerkung über ungerade schlichte funktionen," J. London Math. Soc., [s1-8 (2)], 85-89, 1993.

[13] U. Grenander, G. Szegö, Toeplitz Forms and Their Applications, California Monographs in Mathematical Sciences Univ, California Press, Berkeley, 1958.

[14] H. Ö. Güney, G. Murugusundaramoorthy, J. Sokol, "Subclasses of bi-univalent functions related to shell-like curves connected with Fibonacci numbers," Acta Univ. Sapientiae Mathematica, 10, 70-84, 2018.

[15] T. Hayami, S. Owa, "Coefficient bounds for bi-univalent functions," Pan Amer. Math. J., 22, 15-26, 2012.

[16] I. B. Jung, Y. C. Kim, H. M. Srivastava, "The Hardy space of analytic functions associated with certain one-parameter families of integral operators," J. Math. Anal. Appl., 176, 138-147, 1993.

[17] Y. Komatu, " On analytic prolongation of a family of operators," Math. (Cluj), 32, 141-145, 1990.

[18] M. Lewin, "On a coefficient problem for bi-univalent functions," Proc. Amer. Math. Soc., 18, 63-68, 1967.

[19] R. J. Libera, "Some classes of regular univalent functions," Proc. Amer. Math. Soc., 16, 755-758, 1965.

[20] W. C. Ma, D. Minda, "A unified treatment of some special classes of univalent functions," in: Proceedings of the Conference on Complex Analysis, Tianjin, 1992, pp. 157-169, Conf. Proc. Lecture Notes Anal. I, Int. Press, Cambridge, MA, 1994.

[21] E. Netanyahu, " The minimal distance of the image boundary from the origin and the second coefficient of a univalent function in $|z|<1$," Arch. Rational Mech. Anal., 32, 100-112, 1969.

[22] C. Pommerenke, Univalent Functions, Vandenhoeck \& Ruprecht, Göttingen, 1975.

[23] G. S. Sălăgean, "Subclasses of univalent functions," in: Complex Analysis-Fifth Romanian Finish Seminar, Bucharest, 1983, pp. 362-372.

[24] H. M. Srivastava, S. Sümer Eker, S. G. Hamidi, J. M. Jahangiri, "Faber polynomial coefficient estimates for Bi-univalent funnctions defined by the Tremblay fractional derivative operator," Bull. Iran. Math. Soc., 44, 149-157, 2018.

[25] H. M. Srivastava, A. K. Mishra, P. Gochhayat, "Certain subclasses of analytic and bi-univalent functions," Appl. Math. Lett., 23, 1188-1192, 2010.

[26] H. M. Srivastava, F. M. Sakar, H. Ö. Güney, "Some general coefficient estimates for a new class of analytic and bi-univalent functions defined by a linear combination," Filomat, 32, 1313-1322, 2018. 